\title{
Three-dimensional cylindrical truss structures: a case study for topological optimization
}

\author{
P. Dewhurst, D. G. Taggart, R. B. Waterman \& S. Heinemann \\ College of Engineering, University of Rhode Island, United States
}

\begin{abstract}
A general minimum-weight cylindrical structural layout for the support of any combination of axial and torsional loading has been developed. The principal intention in this work is to provide a test case for 3-dimensional numerical topological optimization. It is anticipated that the solution may present a challenge, since for the small angular spacing of the truss elements the internal radial force component is always of the order of magnitude of the angular spacing for any arbitrary selected pair of helix families. Moreover for a wide range of solutions slender members are an essential part of the topology.

A novel finite element topology optimization procedure is presented based on the application of Beta probability density and cumulative distribution functions. The procedure utilizes a family of Beta functions which provide a smooth transition from a uniform to a bi-modal density distribution, with constant probability mean to conserve constant mass.
\end{abstract}

Keywords: Michell structure, topological optimization, beta function.

\section{Introduction}

Following the initial pioneering work of Michell [1] and a flourish of advances in the 1950's and 1960's (Cox [2]; Hemp [3]; Prager [4]; Chan [5]), the analysis of absolute minimum-weight truss structures entered a period of relative neglect until the 1990's since when it has become a subject of renewed interest; see for example the work by Rozvany et al. [6-8]; Lewinski et al. [9]; Lewinski and Rozvany [10-12]; Graczykowski and Lewinski [13-15]; Dewhurst [16,17]; Dewhurst and Srithongchai [18]; Srithongchai and Dewhurst [19]; Dewhurst et al. [20]. 
The theoretical base for all of the above-mentioned body of work was established by Michell [1] who recognized that, in general, truss structures become more efficient as the spacing of the joints or nodes decrease, and so the theoretical optimum is an infinitely-dense but discrete system of tension and compression members. The members can thus curve continuously, supported by a continuum of connections with other intersecting truss members, forming a paradoxical discrete-continuous network. This allowed Michell to conjecture, and then prove, that an optimal truss must follow the orthogonal network of lines of maximum and minimum strain in a constant-magnitude strain field. The problem of determining the positions of the nodes of an optimal truss design was thus reduced to finding an orthogonal network of layout curves on which the nodes should lie.

The renewed interest in Michell structures has resulted from developments of new methods for numerical structural optimization and the consequent need for proven theoretically-optimal test cases. Pioneering work in this area involved the development of specialized variational principle and material volume constraint equations (Bendsoe and Kikuchi [21]; Bendsoe and Sigmund [22]). This work has great fundamental value since it establishes absolute limits on structural weight. The perceived shortcomings are complexity of the method, and the infeasibility of manufacturing resulting geometries, which often comprise porous regions. In response to the former, a much simpler procedure proposed by Xie and Steven [23,24], was based on progressive irreversible removal of material from low strain energy regions. This method unfortunately has slow convergence, and in some cases fails to find known optimal structural geometries (Zhou and Rozvany [25]). A third methodology, based on iterative adjustment of density and stiffness in finite-element iterations recognizes the 'cost' of design realization of low-density porous regions, and assigns disproportionate stiffness reductions to reduced density. This method, described as solid, isotropic microstructure with penalty (SIMP) by Rozvany et al. [26], has been shown to provide good matching of numerical results to known analytical optimal solutions, and to date SIMP is the only method to be offered commercially.

One singular exception, to the finite element based schemes described above, is a method recently proposed by Martinez et al. [27], which expands an initial simplest starting structure by iteratively dividing structural members, and at each step applying an optimization procedure to reposition the structure nodes for least weight. This method is more narrowly focused but displays remarkable convergence to known Michell solutions.

An alternative finite element based topology optimization scheme is described briefly in this paper (Taggart and Dewhurst [28,29]). This procedure is based on an iterative prescribed material redistribution scheme in which the desired material distribution at each iteration is imposed directly. A family of Beta probability density functions is utilized to provide a gradual transition from an initial unimodal material density distribution to a bimodal distribution of fully dense and essentially void regions. The efficiency and validity of the scheme has been demonstrated through a number of 2-D and 3-D test cases for which the 
optimal topology is known. These test cases include classical minimum weight Michell structures as well as newly derived optimal topologies for 3-D structures. In the present work the results of application of this method to general cylindrical structures is described. The described method is the subject of international patent application number PCT/US2006/062302.

Up to the present time most of the work with both Michell structures and numerical optimization has been two-dimensional. However, a principal focus of the current research project at URI is the investigation of optimal threedimensional structures, and this gives rise to the need for a non-trivial Michell solution to act as a test case. For this purpose, in the present work, the general case of least-weight cylindrical structures for arbitrary combinations of torsional and axial loading is investigated.

Before developing the general cylindrical structural form, it is useful to review the conditions for optimality of a structure which has equal material properties in tension and compression. Michell's original conditions for optimality require that the structure layout must follow the lines of principal strain field in a strain filed with maximum and minimum strains of equal and constant magnitude. The deformation of the strain filed must be compatible with all of the kinematic behavior of the structure, and of course the structure must be in equilibrium. Michell did not stress the latter requirement since his examples were mainly planar and internally statically determinate. His only non-planar structure is a spherical loxodrome solution which is readily recognized to be in equilibrium due to the symmetry of members and forces surrounding each node.

For the case considered here of axial and torsional loading of cylindrical structures, any pair of families of mutually orthogonal helical strain fields are candidates for optimal structure layout, subject to compatible end conditions. However, for given values of axial and torsional loading, only one member of the candidate pairs of families defines an equilibrium structure; which must therefore be the least weight solution. One final comment is in order before proceeding to derive the structural layouts. Since the strain fields are defined on a principal cylindrical surface, the solutions are only shown to be optimal with respect to alternative structures which do no lie outside of the cylindrical envelope.

\section{General cylindrical helical structures}

In the case of pure torsion of a cylinder the lines of maximum and minimum strain follow opposite families of 45-degree helices. The truss structure formed by placing nodes, at constant angular increments along these helices, whose connecting members are equal helical chords, thus satisfies all of the requirements of a least-weight Michell structure. A rapid prototyped example of such a structure is shown in Fig. 1.

In this case nodes are positioned at 45-degree angular increments around the cylinder. Pure torsion introduces the same force magnitude into the tension and compression members, and the symmetry of the members surrounding each node ensures that the structure is in equilibrium. In particular the radially inward 
component of the two tension members at each node is cancelled by the radially outward component of the two compression members. If an axial load is introduced in addition to the torsion load, then tension and compression members will carry different internal forces and radial equilibrium will no longer exist. In this case the structure will be globally unstable at only a fraction of the design load. The general requirement for radial equilibrium will be examined next.

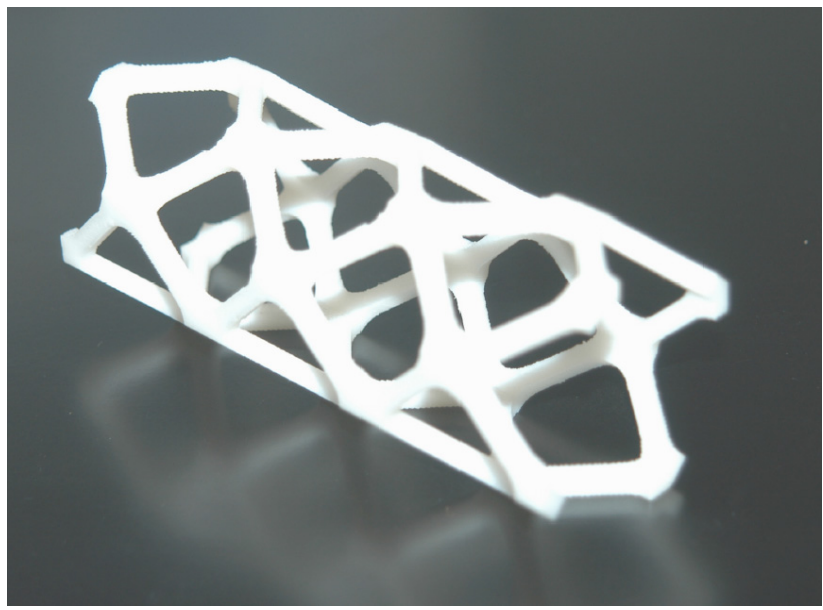

Figure 1: $\quad$ Pure torsion cylindrical structure.

\subsection{Condition for radial equilibrium}

The combination of axial and torsional loading of course gives rise to principal strain directions which follow two orthogonal families of opposite equi-angular helices. Below we will examine this more general strain field to determine the conditions for equilibrium with arbitrary ratios of axial to torsional loading.

Consider two families of equi-angular helices; left-hand $\alpha$ helices intersecting the axial direction at constant angle $\gamma$, and right-hand $\beta$ helices intersecting the axial direction at constant angle $(\pi / 2-\gamma)$. It can be shown that the $\alpha$ helices are represented by the parametric equations

$$
x=r \cos (\theta) ; y=-r \sin (\theta) ; z=r \cot (\gamma) \theta
$$

and the $\beta$ helices by

$$
x=r \cos \left(\theta^{\prime}\right) ; y=r \sin \left(\theta^{\prime}\right) ; z=r \tan (\gamma) \theta^{\prime}
$$

where $\theta$ and $\theta^{\prime}$ are angles of rotation about the z-axis measured counterclockwise and clockwise respectively from the x-axis. Without loss of generality the $\alpha$ helices will be taken as the layout lines for the tension members and the $\beta$ helices will lay out the compression ones. 


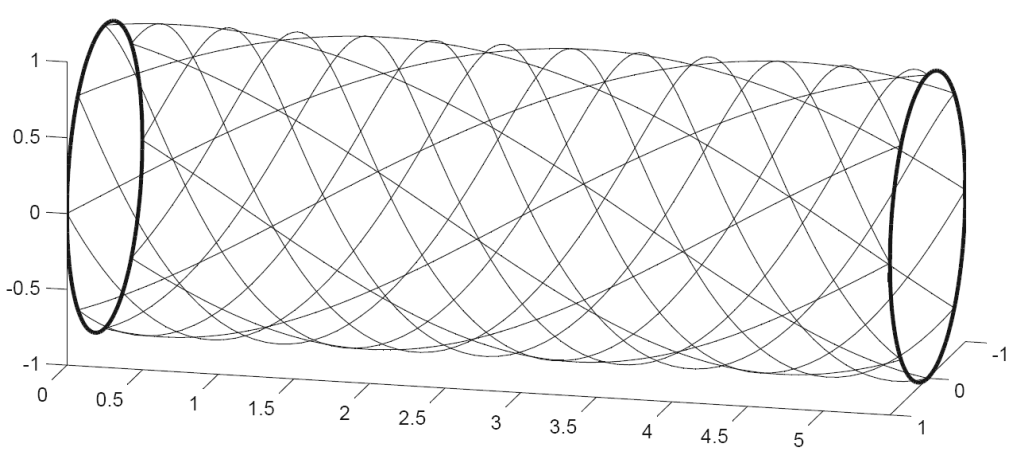

Figure 2: $\quad$ Strain field for axial and torsional loading $(\gamma=\pi / 6)$.

Figure 2 illustrates the helical network produced by the two helical families for the case $\gamma=\pi / 6$.

To establish the relationship between $\theta$ and $\theta^{\prime}$ between the nodes of the helical network we can note that they involve equal separation in the axial direction $(z)$ along the two families. Thus from (1) and (2)

$$
r \cot (\gamma) \theta=r \tan (\gamma) \theta^{\prime} \text { or } \theta^{\prime}=\cot ^{2}(\gamma) \theta
$$

Michell structures may be considered as truss structures with closely spaced nodes so the discrete members follow as closely as possible the curvilinear optimal strain field. For the present case we will consider the nodes to be separated by constant parameter increments $\Delta \theta$ and $\Delta \theta^{\prime}$ respectively. The members connected an arbitrarily selected node, taken as a local origin, thus have co-ordinate lengths given by Eqs. (1) and (2) as

$$
\begin{array}{r}
\Delta x=r \Delta \theta^{2} / 2 ; \Delta y=r \Delta \theta ; \Delta z=r \cot (\gamma) \Delta \theta \\
\Delta x=r \Delta \theta^{\prime 2} / 2 ; \Delta y=r \Delta \theta^{\prime} ; \Delta z=r \tan (\gamma) \Delta \theta^{\prime}
\end{array}
$$

for the tension and compression members respectively. Assume the tension members carry internal force $f_{T}$ and the compression members force $f_{C}$. Equations (4) and (5) show that the tension and compression members are orthogonal in the $\mathrm{y}-\mathrm{z}$ plane to order $\Delta \theta^{2}$ and so equilibrium in the $\mathrm{y}$ and $\mathrm{z}$ directions is assured. It therefore only remains to establish equilibrium in the $\mathrm{x}$ direction. From Eqs.(4), the component of tensile force $f_{T}$ in the x-direction is given by

$$
f_{T x}=f_{T}\left[\frac{\Delta \theta}{2\left(1+\cot ^{2}(\gamma)\right)^{1 / 2}}\right]
$$

where the term in brackets is the $\mathrm{x}$-component of the unit vector along the tensile member. 
Similarly from Eqs.(5) the x-component of compressive force $f_{C}$ is

$$
f_{C x}=f_{C}\left[\frac{\Delta \theta^{\prime}}{2\left(1+\tan ^{2}(\gamma)\right)^{1 / 2}}\right]
$$

and substituting for $\theta^{\prime}$ from Eq.(3) gives

$$
f_{C x}=f_{C}\left[\frac{\cot ^{2}(\gamma) \Delta \theta}{2\left(1+\tan ^{2}(\gamma)\right)^{1 / 2}}\right]
$$

Substituting Eqs.(6) and (8) into the required condition $f_{T x}=f_{C x}$ gives after some trigonometric manipulation

$$
f_{C}=\tan ^{3}(\gamma) f_{T}
$$

\subsection{Internal forces in structural members}

A necessary step to establish the truss volume is to relate the equilibrium condition, and the axial and torsional loads imposed on the structure, to the internal forces in the structural members. Figure 3 shows the axial and shear force components imposed on a single node from the external loading. Applying axial and circumferential equilibrium conditions on the node, and

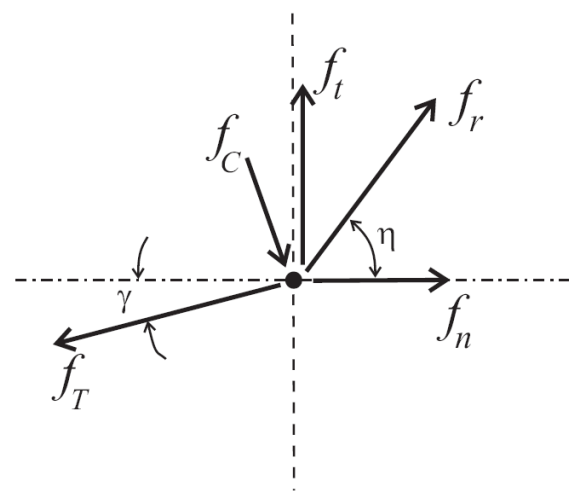

Figure 3: Forces acting on node.

substituting for $f_{C}$ from radial equilibrium condition (9) gives

$$
\begin{aligned}
& f_{n}=f_{T}\left(\cos (\gamma)-\sin ^{4}(\gamma) / \cos ^{3}(\gamma)\right) \\
& f_{t}=f_{T}\left(\sin (\gamma)+\sin ^{3}(\gamma) / \cos ^{2}(\gamma)\right)
\end{aligned}
$$

Dividing Eqs.(14) and simplifying leads to the elegant result

$$
\gamma=\cot ^{-1}\left(\left(f_{n} / f_{t}\right) / 2\right) / 2=\cot ^{-1}(\cot (\eta) / 2) / 2
$$

where $\eta$ is the angle of the resultant force; see Fig.3. 
This result ranges from $\gamma=\pi / 4$ for $f_{n}=0$ (pure torsion) to $\gamma=0$ for $f_{t}=0$ (pure axial loading. For the latter, the structure has become simply a set of axial members at angular spacing $\Delta \theta$ on a pitch circle of radius $r$. Note also at this point Eq.(9) predicts $f_{C}=0$ consistent with the circular column limiting solution.

In terms of the global loading on the structure, the axial force $F$ and torque $T$ are represented by

$$
F=2 \pi f_{n} / \Delta \phi ; T=2 \pi r f_{t} / \Delta \phi
$$

where $\Delta \phi=\Delta \theta+\Delta \theta^{\prime}$ is the angular spacing of nodes around the cylinder.

Thus for any arbitrary combination of axial load $F$ and torque $T$, the optimal cylindrical structure layout comprises orthogonal families of helices intersecting the axial direction at angles $\gamma$ and $(\pi / 2-\gamma)$ respectively, where

$$
\gamma=\cot ^{-1}((F r / T) / 2) / 2
$$

\section{Prescribed material redistribution method}

A finite element based topology optimization procedure has been developed in which, through a series of iterative analyses, material is redistributed according to prescribed distributions of nodal densities. This procedure has been named the "prescribed material redistribution" (PMR) method. In this procedure, the desired final mass of the structure is specified at the beginning of the analysis. This material mass is initially distributed uniformly throughout the design domain resulting in a uniform, partially-dense material. All nodes are assigned an initial relative density $\rho_{o}=V_{f} / V_{D}$, where $V_{f}$ is the final structural volume and $V_{D}$ is the volume of the partially-dense design domain. This initial distribution can be described by the probability distribution function, $f_{o}$, given by $f_{o}(\rho)=\delta\left(\rho-\rho_{0}\right)$, where $\delta$ is the Dirac delta function and $\rho$ is the relative material density $(0 \leq \rho \leq 1)$. The corresponding cumulative distribution function, $F_{o}$, is given by $F_{o}(\rho)=H\left(\rho-\rho_{0}\right)$, where $H$ is the Heaviside step function. The desired final material distribution contains two distinct regions of fully dense material $(\rho=1)$ and regions that have zero relative density $\left(\rho_{\min }<<1\right)$ with the fully dense regions representing the optimized topology. The final material distribution can be described by the probability distribution function, $f_{f}$, given by

$$
f_{f}(\rho)=\left(1-\rho_{o}\right) \delta\left(\rho-\rho_{\min }\right)+\rho_{o} \delta(\rho-1)
$$

and the corresponding final cumulative distribution is given by

$$
F_{f}(\rho)=\left(1-\rho_{o}\right) H\left(\rho-\rho_{\min }\right)+\rho_{o} H(\rho-1)
$$

A gradual transition from the initial distribution to the final distribution can be achieved through the use of the beta function

$$
f(\rho)=\beta(\rho, r, s)=\frac{\rho^{r-1}(1-\rho)^{s-1}}{B(r s)}
$$


where $r$ and $s$ are adjustable parameters and

$$
B(r, s)=\frac{\Gamma(r) \Gamma(s)}{\Gamma(r+s)}
$$

where $\Gamma$ is the gamma function. The corresponding cumulative distribution function, also known as the incomplete beta function, is given by

$$
F(\rho)=\beta_{i n c}(\rho, r, s)=\frac{1}{B(r, s)} \int_{0}^{\rho}\left(\rho^{\prime}\right)^{r-1}\left(1-\rho^{\prime}\right)^{s-1} d \rho^{\prime}
$$

A non-dimensional time parameter, $t$, where $0 \leq t \leq 1$, is introduced and appropriate functions $r(t)$ and $s(t)$ are specified. The functions $r(t)$ and $s(t)$ are selected such that the total mass of material is held constant and a smooth transition from the initial unimodal distribution to the final bimodal distribution is achieved. One such family of distributions are shown in Fig. 4.

At each finite element iteration, nodal densities are assigned based on the sorted nodal strain energies computed from the previous iteration. Nodes with relatively low strain energy are assigned reduced nodal densities and nodes with relatively high strain energy are assigned increased nodal densities. Using a prescribed assignment of nodal densities, the desired progression of density distributions is enforced. In computing the element stiffness matrices, the nodal density field is interpolated to give the Young's modulus, $E$, at each Gauss point according to the relation $E=E_{d} \rho$ where $E_{d}$ is the fully dense Young's modulus. Convergence to the final topology can be achieved in relatively few finite element iterations.
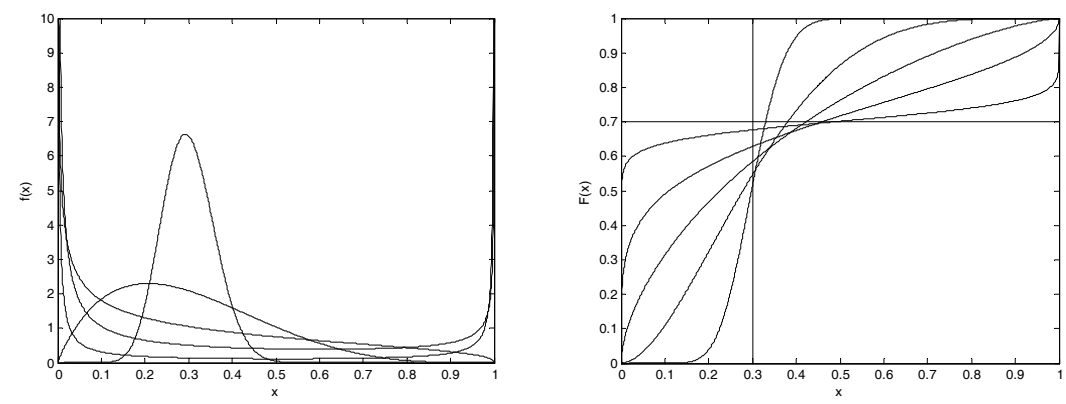

Figure 4: Beta distributions (density and cumulative) showing transition from initial to final distribution for $\mathrm{t}=0.1,0.3,0.5,0.7$, and 0.9 .

\subsection{Case studies}

The numerical procedure has been shown to converge rapidly to a wide range of known two-dimensional optimal truss structures. The case shown in Fig. 5 is for a load at the mid-point of a pinned and a roller support. The selected design domain is shown on the left of the figure and the resulting topology on the right is the well known center fan topology obtained by Michell [1]. 
For the present problem, the design domain is taken to be a thick walled cylinder subjected to concentrated forces at the ends of the cylinder given by $f_{n}$ in the axial and $f_{t}$ in the tangential directions. The optimized results for cases ranging from pure axial loading $\left(f_{n}=1, f_{t}=0\right)$ to pure torsion $\left(f_{n}=0, f_{t}=1\right)$ are shown in Fig. 6, together with the effect of decreasing element size.

The helix angle, $\gamma$, was estimated from these models and compared to the theoretical optimum given in Equation 17. As shown in Figure 7, the numerical
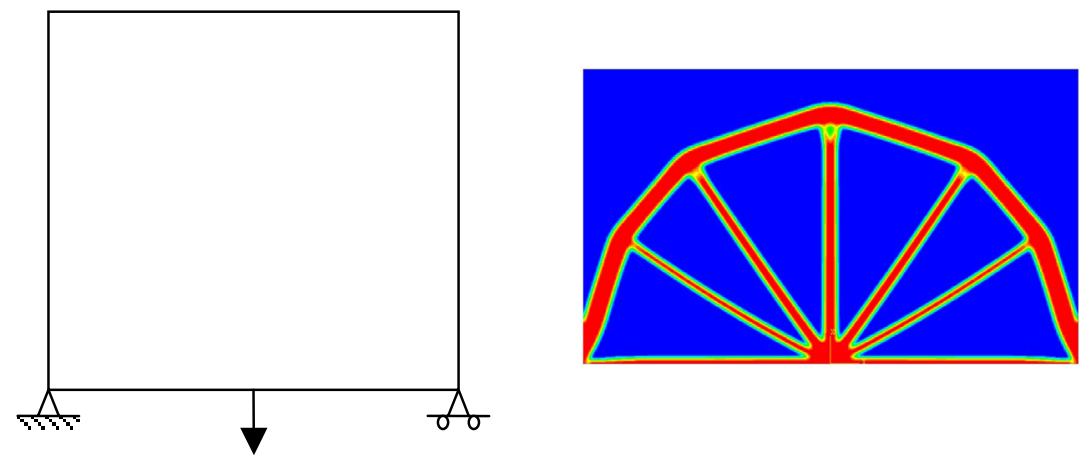

Figure 5: $\quad$ 2-D domain and resulting topology.
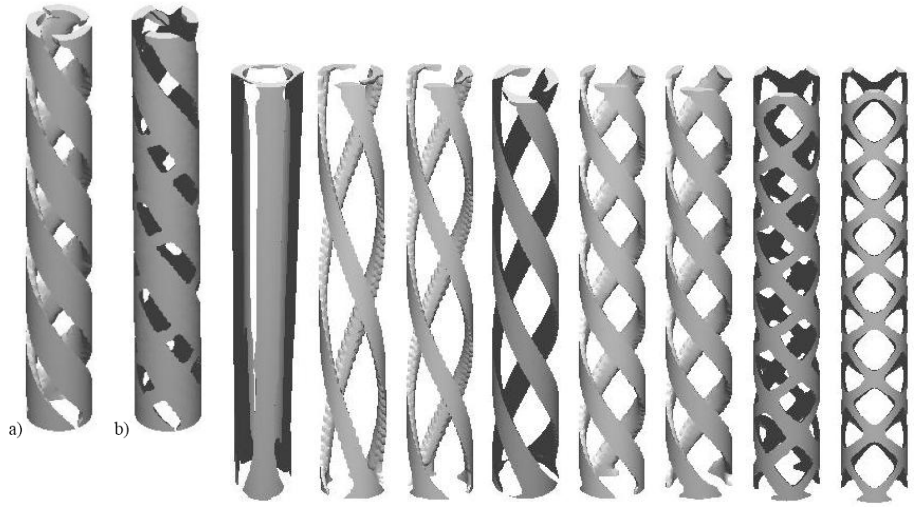

Figure 6: Results for combined axial and torsion loads; including effect of increasing number elements from (a) 5452 to (b) 19584 for $\left(f_{n} / f_{t}=1\right)$.

scheme accurately predicts the optimum helix angle for all cases. It should be noted that pronounced divergence exists between the helix angles, $\gamma$, and the angle of the resultant forces, $\eta$, being applied to the boundary. The difficulty of the case, for topology optimization, is that a single set of members tends to propagate from the boundary from the resultant forces, but then follow the optimal helix angle. Producing the complimentary set of supporting helical members is a challenge. 


\section{Discussion}

A generalized minimum-weight cylindrical structural layout for the support of any combination of axial and torsional loading has been developed. The principal intention in this work is to provide a challenging test case for 3dimensional numerical topological optimization. The case presents two difficulties for topological optimization. The optimal topology is determined by the condition of radial equilibrium. However from Eqs. (6) and (7) it can be seen that any non-equilibrated radial force, resulting from a non-optimal helix angle $\gamma$, will only be of the order of magnitude of the node angular separation. The second difficulty stems from the fact that the ratio of the internal forces in the compression and tension helical members is equal to $\tan ^{3} \gamma$. Thus for $\gamma<25^{\circ}$ or $\gamma>65^{\circ}$ one of the helical families will carry less than $10 \%$ of the internal load of the other family. Getting both sets of helical members to emerge proved to be a challenge for NMR, as seen in Fig. 6. The authors would be interested in any attempts by interested readers to produce the full family of solutions by SIMP or other methods.

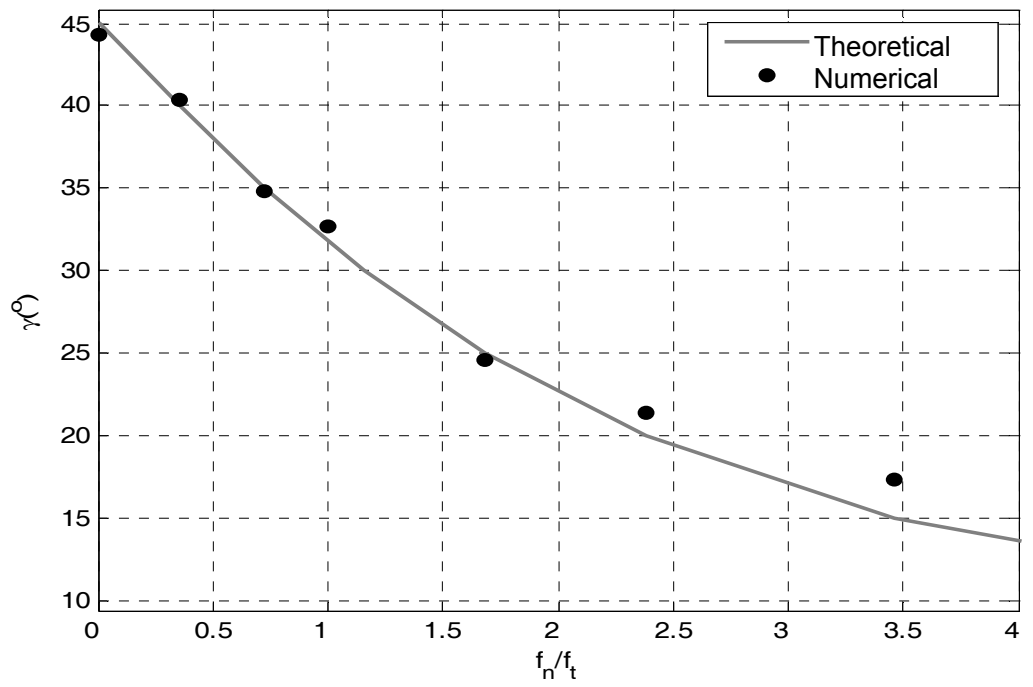

Figure 7: Comparison of theoretical and numerical optimum helix angle $\gamma$.

\section{Acknowledgements}

This material is based upon work supported by the National Science Foundation under Collaborative Research Grant Nos. 0620752 and by Sandia National Laboratories, Albuquerque, New Mexico. 


\section{References}

[1] Michell AGM, The limits of economy of material in frame structures. Phil Mag 8, pp. 589-597, 1904.

[2] Cox HL, The Theory of design. Aeronautical Research Council 19791, 1958.

[3] Hemp WS, Theory of the structural design. The College of Aeronautics, Cranfield. Report No.115, 1958.

[4] Prager W., A problem of optimal design. Proceedings of the Union of Theoretical and Applied Mechanics, Warsaw, 1958.

[5] Chan ASL, The design of Michell optimum structures. The College of Aeronautics, Cranfield. Report No. 142, 1960

[6] Rozvany GIN, Gollub W, Zhou M, Exact Michell layouts for various combinations of line supports. Part II. Struct Optim 14, pp.138-149, 1997.

[7] Rozvany GIN, Bendsoe MP, Kirsch U, Layout optimization of structures. Appl Mech Rev 48, pp.41-118, 1995.

[8] Rozvany GIN, Querin OM, Logo J, Pomezanski V, Exact analytical theory of topology optimization with some pre-existing members or elements. Struct Multidisc Optim 31, pp.373-377, 2006.

[9] Lewinski T, Zhou M, Rozvany GIN, Extended exact solutions for leastweight truss layouts. Part I. Cantilever with a horizontal axis of symmetry. Part II. Unsymmetric cantilevers. Int J Mech Sci 36, pp.375-419, 1994.

[10] Lewinski T, Rozvany GIN, Exact analytical solutions for some popular benchmark problems in topology optimization II: Three-sided polygonal supports. Struct Multidisc Optim 33, pp.337-349, 2007.

[11] Lewinski T, Rozvany GIN, Exact analytical solutions for some popular benchmark problems in topology optimization III: $L$-shaped domains. Struct Multidisc Optim 35, pp.165-174, 2008.

[12] Lewinski T, Rozvany GIN, Exact analytical solutions for some popular benchmark problems in topology optimization IV: square-shaped line support. Struct Multidisc Optim 36, pp.143-158, 2008.

[13] Graczykowski, C, Lewinski T, Michell cantilevers constructed within trapezoidal domains. Part I. Geometry of Hencky nets. Struct Multidisc Optim 32, pp.347-368, 2006.

[14] Graczykowski, C, Lewinski T, Michell cantilevers constructed within trapezoidal domains. Part III: Force fields. Struct Multidisc Optim 33, pp.119, 2007.

[15] Graczykowski, C, Lewinski T, Michell cantilevers constructed within trapezoidal domains. Part IV: Complete exact solutions of selected optimal designs and their approximations by trusses of finite number of joints. Struct Multidisc Optim 33, pp.113-129, 2007.

[16] Dewhurst P, Analytical solutions and numerical procedures for minimumweight Michell structures. J Mech Phys Solids 49, pp.445-467, 2001.

[17] Dewhurst P (2005) A general optimality criterion for strength and stiffness of dual-material-property structures. Int. J. Mech. Sci. 47, pp.293-302, 2005. 
[18] Dewhurst P, Srithongchai S, An investigation of minimum-weight dual material symmetrically loaded wheels and torsion arms. J of App Mech 72, pp.196-202, 2005.

[19] Srithongchai S, Dewhurst P, Comparisons of optimality criteria for minimum-weight dual material structures. Int J Mech Sci 45, pp.1781-1797, 2003.

[20] Dewhurst P, Fang N, Srithongchai S A general boundary approach to the construction of Michell truss structures. Struct Multidisc Optim (in press)

[21] Bendsoe MP, Kikuchi N, Generating optimal topologies in structural design using a homogenization method. Comput Meth Appl Mech Engrg. 71, pp.197-224, 1988.

[22] Bendsoe MP, Sigmund O, Topology optimization: theory, methods and applications. Springer-Verlag, Berlin, 2004.

[23] Xie YM, Steven GP, A simple evolutionary procedure for structural optimization. Comput Struct 49, pp.885-896, 1993.

[24] Xie YM, Steven GP, Evolutionary structural optimization. Springer, London, 1997.

[25] Zhou M and Rozvany GIN, On the validity of ESO type methods in topology optimization, Struct Multidisc Optim 21, pp.80-83, 2000.

[26] Rozvany GIN, Zhou, M, Birker, T, Generalized shape optimization without homogenization. Struct. Optim. 4, pp.250-252 (1992).

[27] Martinez P, Marti P, Querin OM, Growth method for size, topology, and geometry optimization of truss structures. Struct Multidisc Optim 33, pp.1326, 2007.

[28] Taggart DG, Dewhurst P, A novel topological optimization method. $8^{\text {th }}$ World Congress on Computational Mechanics (WCCM8), Venice , Italy, 2008.

[29] Taggart DG, Dewhurst P, A finite element based topology optimization scheme based on prescribed material redistribution. Struct Multidisc Optim (in review), 2009. 